\title{
In Vivo Diffusion Tensor Imaging of Chronic Spinal Cord Compression in Rat Model
}

\author{
Matthew M. Cheung, Darrell T.H. Li, Edward S. Hui, Shujuan Fan, \\ Abby Y. Ding, Yong Hu and Ed X. Wu*
}

\begin{abstract}
Chronic spinal cord compression induced cervical myelopathy is a comon cause of spinal cord dysfunction. The exact mechanisms of underlying progressive cell death remain to be elucidated. In this study, in vivo diffusion tensor imaging (DTI) has been applied to investigate the microstructural changes of white matter (WM) in this neurodegenerative disease. Compared with conventional MRI techniques, DTI is believed to be more specific to pathological changes. Radial diffusivity $\left(\lambda_{\perp}\right)$ is higher in the ipilesional region, suggesting demyelination or axonal degradation may occur after prolonged compression. Near the epicenter of lesion, axial diffusivity $(\lambda / /)$ is lower. Also, caudal-rostral asymmetry has been observed in $\lambda / /$. Feasibility of using DTI to detect microstructural changes in chronic disease has been demonstrated.
\end{abstract}

Index Terms - Cervical myelopathy, MRI, DTI, spinal cord compression, axial diffusivity, radial diffusivity

\section{INTRODUCTION}

$\mathrm{C}$ hronic spinal cord compression induced cervical myelopathy is the most commonly acquired cause of spinal cord dysfunction. The neurodegenerative disease leads to impairment of motor and sensory functions progressively [1]. Age related changes such as drift of veterbral bodies, infolding of ligamentum flavum, formation of osteophytes cause an extrinsic pressure on the spinal cord [2]. The static and dynamic factors trigger various changes such as ischemia, excitotoxicity and apoptosis $[2,3]$. It is believed that vascular factors play an important role in the pathogenesis of myelopathy [2], yet the precise mechanism of how the disease progresses remains to be investigated $[1,2]$. In order to understand the mechanisms and assess treatment efficacy, non-invasive imaging technique is essential. In this study, a rat model with chronic spinal cord compression was examined by in vivo diffusion tensor imaging (DTI) to investigate the nature of neurodegeneration of this disease.

Manuscript received April 7, 2009. This work was supported in part by the University of Hong Kong CRCG grant and Hong Kong Research Grant Council grant.

Ed X. Wu, Matthew M. Cheung, Edward S. Hui, Abby Y. Ding and Shujuan Fan are with the Laboratory of Biomedical Imaging and Signal Processing and the Department of Electrical and Electronic Engineering, The University of Hong Kong, Pokfulam, Hong Kong (phone: (852) 2819-9713; e-mail: ewu@eee.hku.hk). Darrell T. H. Li and Yong Hu are with Department of Orthopaedics and Traumatology, The University of Hong Kong, Pokfulam, Hong Kong.
Compared with conventional MRI techniques, DTI has been demonstrated to be more pathologic specific as it provides detailed information about tissue microstructure [4]. MR diffusion has been shown to be sensitive in probing microstructural changes in different pathophysiological situations [5-9]. DTI-derived indices reflecting diffusion anisotropy and directional diffusivities are useful to reveal fiber integrity. Reduction in diffusion anisotropy is believed to reflect the structural changes in white matter (WM) fibers [4]. It has been verified that pathologic specificity is improved with analysis of individual eigenvalues of the DT [10].

Both in vivo [4, 11, 12] and ex vivo [13-15] DTI has been employed to investigate white matter integrity in different spinal cord disorders. These studies focus on acute spinal cord injury or multiple sclerosis, and the results have shown great promise in detecting microstructural alterations. In these studies, DTI indices have been demonstrated to be sensitive markers of white matter pathology. Due to the need of high spatial resolution, many of the studies were performed on excised rodent spinal cords. However, diffusivities and anisotropy of fixed spinal cords have been known to be lower than that from the in vivo situation. In these in vivo studies, RF coil has been implanted for image quality improvement. Although SNR can be improved when implantable coil is used instead of external coil, reproducibility of the practical steps in setting up the coil must be ensured carefully [16]. This step is essential especially when large number of animals is required in longitudinal studies. Recently, studies without RF coil implantation have shown that it is possible to obtain in vivo diffusion properties of spinal cord at different regions [17, 18]. In this study, we demonstrate the feasibility of using DTI in detecting chronic neurodegeneration, and therefore the potential to study spinal cord injury or disease longitudinally by DTI is possible in the future.

\section{MAterials AND Methods}

\section{A. Animal Preparation}

Under anesthesia, laminae of $\mathrm{C} 3-\mathrm{C} 7$ of 5 adult Sprague Dawley rats $(250-300 \mathrm{~g})$ were exposed carefully. A urethane containing polymer was then inserted into the lateral column of C5-C6 and allowed to expand upon absorbing tissue fluid for 9 months before MR acquisition. The polymer can 
expand up to $300 \%$ of its original size and it exerts a compressive force onto the spinal cord.

\section{B. MR acquisition}

Animals were anesthetized with a mixture of isoflurane/air at $3 \%$ for induction and $1.5 \%$ for maintenance throughout the scan. A warming pad with circulating water was used throughout the experiment. MR acquisition was performed with a Bruker Pharmascan 7T scanner, with a birdcage transmit-only coil with a $72 \mathrm{~mm}$ inner diameter in combination with an actively decoupled receive-only quadrature surface coil. A respiratory sensory pillow was placed on the abdomen for gating. 4-shot echo planar imaging sequence with the implementation of navigator echo for correcting N/2 ghost,

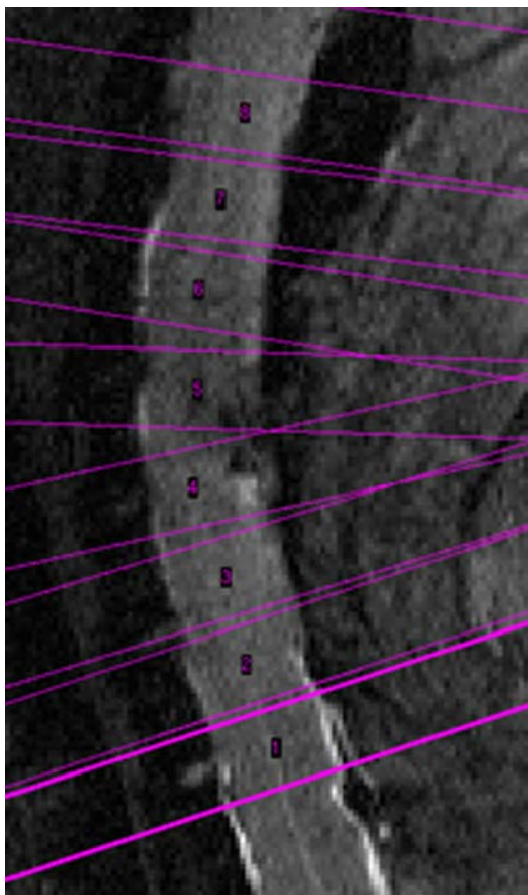

Fig.1 Localization of slices. Slices were oriented perpendicular to the spinal cord. correcting $\mathrm{N} / 2$ ghost, frequency and phase shifts between shots was used with the following parameters: $\mathrm{TR} / \mathrm{TE}=3000 / 29 \mathrm{~ms}$, $\delta / \Delta=3.5 / 17 \mathrm{~ms}, \quad$ slice thickness $=2 \mathrm{~mm}$ with $0.2 \mathrm{~mm}$ inter-slice gap, $\mathrm{FOV}=30 \mathrm{~mm}, \quad$ data matrix $=128 \times 128$,

$\mathrm{NEX}=4$. Diffusion encoded gradients with $\quad \mathrm{b}=0.8 \mathrm{~ms} / \mu \mathrm{m}^{2}$ were applied along 30 directions.

\section{Data Analysis}

Co-registration of all diffusion weighted (DW) images was performed by AIRv5.2.5 before DT estimation. The DT computation was performed by

DTIStudio (John Hopkins University, Baltimore, Md.), DTI parametric maps, including trace, fractional anisotropy (FA), axial diffusivity $\left(\lambda_{/ /}\right)$and radial diffusivity $(\lambda \perp)$ were exported. The spatial variations of the 4 defined ROIs were compared by two-way ANOVA, followed by Bonferroni's test to detect inter-group differences.

\section{RESULTS}

Localization of slices of a typical animal is shown in Fig. 1. Each slice was oriented to be perpendicular to the cord in scout images. Parametric maps of a slice covering the lesion cite is shown in Fig. 2. The polymer compressed the spinal cord from the left side of the figure. The shape of the spinal cord was deformed and hence the compression was successfully made. The deformed " $\mathrm{H}$ " shaped grey matter is still differentiable from the white matter in this animal. However, in some of the animals (2/5), the compression has severely distorted ipsilesional WM and the area of WM varies significantly. As a result, Regions of interest (ROIs) were not measured at the epicenter of injury. Regions of interest of 4 WM regions (V: ventral, D: dorsal, I: ipsilesional, C:contralesional) in 8 slices covering $4.4 \mathrm{~mm}$ caudal and 4.4 $\mathrm{mm}$ rostral to the epicenter of injury were manually defined on FA and $\lambda_{\perp}$ maps. Typical ROI delineation is shown in Fig. 3. Parameters at different distance with the epicenter of the injury were measured and the spatial variations of them were plotted as in Fig. 3 (red: ipsilesional, blue: dorsal, green: ventral, black: contralesional). The ipsilesional region has a lower FA and higher $\lambda \perp$ than other regions near the lesion site.

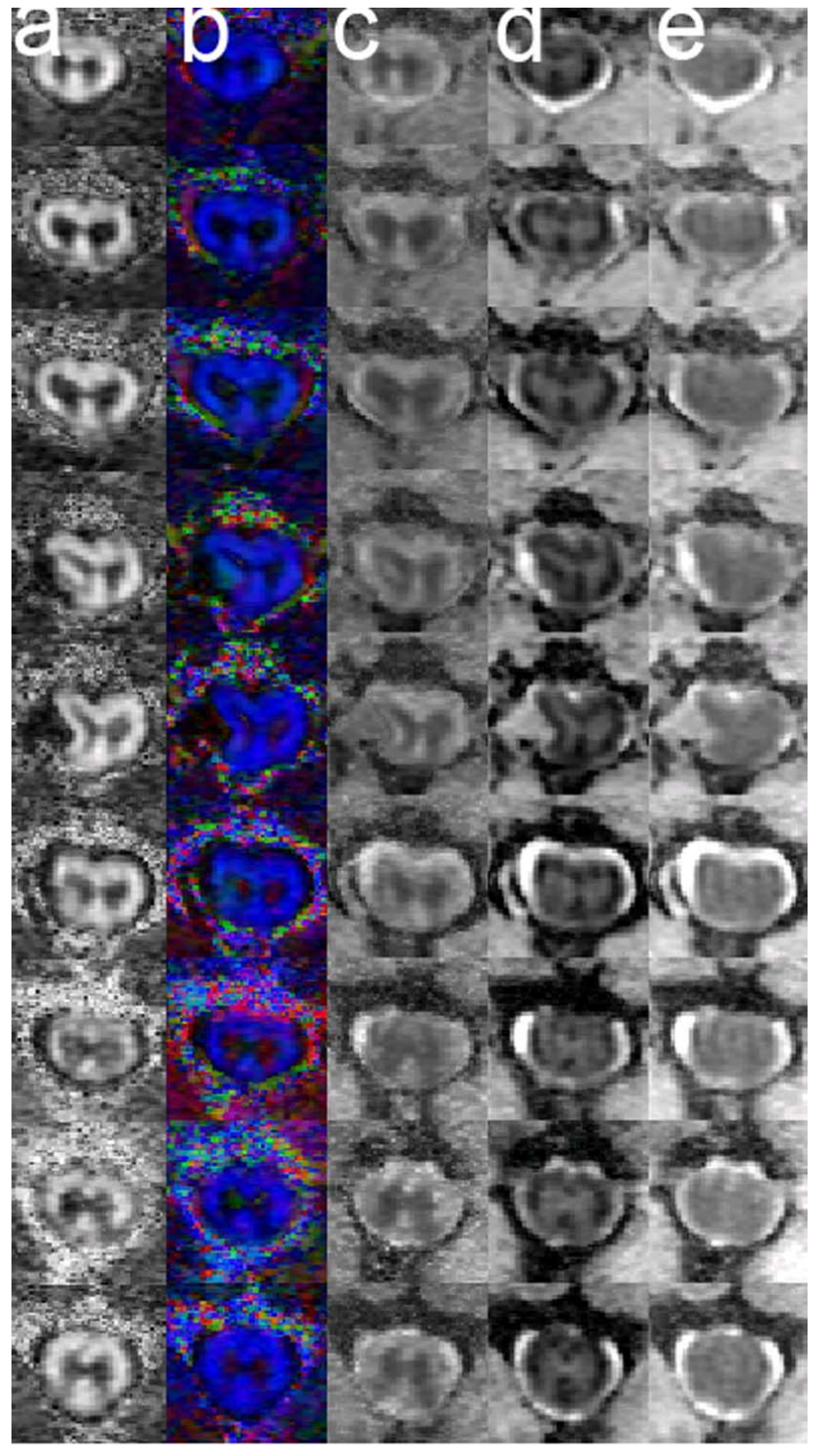

Fig. 2 parametric maps of the 9 slices covering the lesion are a) FA, b) color-coded FA, c) $\lambda_{/ /}$, d) $\lambda_{\perp}$ and e)trace maps 


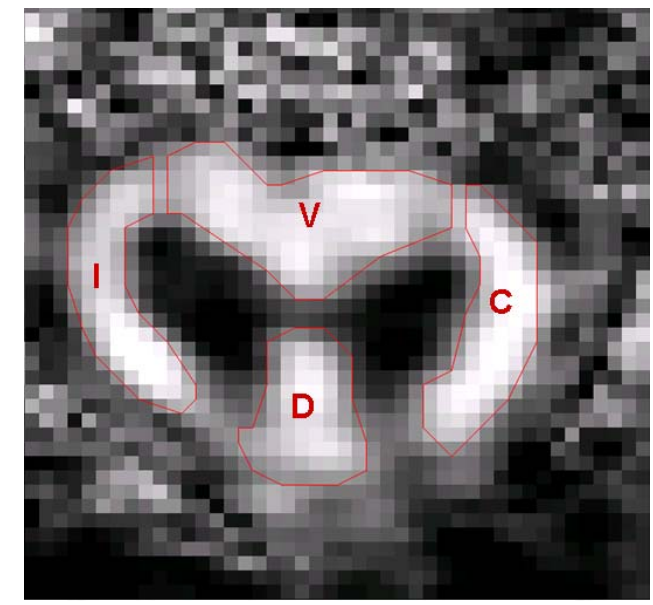

Fig.3 ROI delineation. Four white matter regions were defined: Ventral (V), Dorsal (D), Ipsilesional (I) and C (Contralesional).
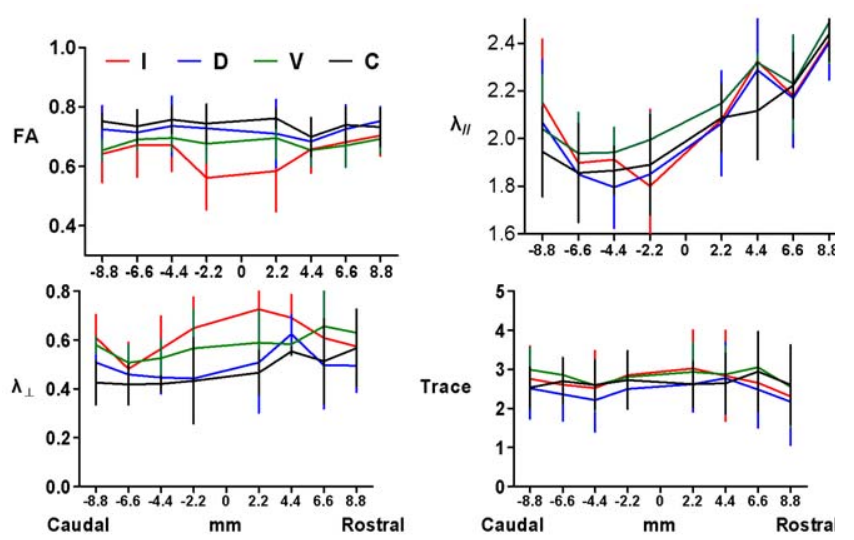

Fig. 4 Average $(\mathrm{N}=5)$ diffusion characteristic along the $\mathrm{SC}(\mathrm{Mean} \pm \mathrm{SD})$. Red: Ipsilesional(I);Blue: Dorsal (D); Green: Ventral (V); Black: Contralesional (C). $\lambda_{/ /}, \lambda \perp$ and Trace are listed in $\mu \mathrm{m}^{2} / \mathrm{ms}$.

\section{DisCUSSIONS}

The chronic spinal cord compression induced cervical myelopathy is characterized by its delayed and progressive temporal characteristics. The precise mechanism is still not well understood yet. The feasibility of DTI to detect microstructural changes in this progressive disease is therefore important for better understanding and diagnosing the disease, and evaluating drug efficacy.

As shown in Fig.2, the water absorbing polymer compressed the spinal cord laterally and the ipsilesional WM was distorted. The injuries to other regions appear to be moderate and such compression did not appear to have significant effect on other WM regions. Additionally, the fibers remain to be aligned coherently as shown in the color-coded FA map in Fig. 2b. The homogeneous blue color in the remaining WM suggests that the principle eigenvectors, and hence the fiber orientation, align longitudinally after the compression.

The spatial variation is shown in Fig. 4. Dorsal, ventral and contralesional regions show a relatively flat curve in all 4 indices. These regions exhibit values of FA and $\lambda_{\perp}$ closer to normal. The spatial variation is statistically significant $(\mathrm{p}<0.05)$ in FA and $\lambda_{\perp}$ among different ROIs. Bonferroni's tests reveal that FA of ipsilesional region is statistically lower $(p<0.05)$ than that of dorsal and contralesional regions near the epicenter of lesion, and $\lambda \perp$ of ipsilesional region is found to be higher than that of contralesional region near the epicenter of lesion $(p<0.05)$. This suggests demyelination may occur after prolonged compression. It is consistent with previous studies about chronic degeneration of WM bundles in humans [19]. In delayed and progressive myelopathy, demyelination has been observed by histology in experiments using dogs or rodents model, however both studies have added that demyelination was not evident $[1,20]$. Demyelination has been shown to modulate $\lambda \perp$ [21], but it is known that myelin is not the only factor [22]. The organization of axons may also have altered also. Degradation of axonal packing might be a factor of altering the anisotropy and diffusivity in the radial direction. Near the epicenter of lesion in the ipsilesional area, $\lambda_{/ /}$is lower $(\mathrm{p}<0.05$ without statistical significant differences among 4 ROIs), axonal damage may also occur there.

It is interesting to see that $\lambda_{/ /}$in the rostral region increases when it is farther away from the epicenter of lesion. Alternatively, the $\lambda_{/ /}$remains similar in the caudal region. A one-tailed paired t-test was then performed retrospectively. Rostral regions of dorsal, ventral and contrlesional sides exhibits higher $\lambda_{/ /}$than caudal $(\mathrm{p}<0.05)$, but the difference is not statiscally significant in rostral region of ipsilesional side $(p=0.102)$. This may suggest that there are more aligned axons in the rostral section of the cord. This result is consistent with a previous study on the electrophysiology of a cat with acute spinal cord compression [23]. Action potential caudal to the lesion site was found to be dimished. The reason of this asymmetry may be due to the more compromised blood flow in the rostral section. The better blood flow in rostral region is hypothesized in a study that investigates how the levels of $\mathrm{N}$-acetyl aspartate (NAA) change in a spinal cord transectomy $[4,24]$. NAA levels were found to be unchanged in the acute stage of recovery. The better blood flow may benefits the axons such that they can be relatively intact in the rostral region. Further studies are warranted to elucidate the elevated level of $\lambda_{/ /}$in the rostral part.

In this study, the polymer was inserted into the spinal cord at lateral side. In clinical situations such as spondylosis and ossification of the posterior longitudinal ligament can be caused by damage from the ventral region, and the location is dorsal in hypertrophied yellow ligament[2]. Therefore, the rodent model may not fully reflect the clinical situation in this study.

\section{CONCLUSION}

We have demonstrated the feasibility of using DTI to investigate the neurodegeneration of the chronic spinal cord compression. The changes of DTI parameters in the lesion are possibly due to the microstructural changes caused by the pathology. In vivo serial DTI is therefore applicable to perform longitudinal study in chronic diseases. 


\section{ACKNOWLEDGMENT}

The work was in part supported by Hong Kong Research Grant Council and HKU CRCG grants.

\section{REFERENCES}

[1] P. Kim, T. Haisa, T. Kawamoto, T. Kirino, and S. Wakai, "Delayed myelopathy induced by chronic compression in the rat spinal cord," Ann Neurol, vol. 55, pp. 503-11, Apr 2004.

[2] R. Rao, "Neck pain, cervical radiculopathy, and cervical myelopathy: pathophysiology, natural history, and clinical evaluation," J Bone Joint Surg Am, vol. 84-A, pp. 1872-81, Oct 2002.

[3] D. C. Baptiste and M. G. Fehlings, "Pathophysiology of cervical myelopathy," Spine J, vol. 6, pp. 190S-197S, Nov-Dec 2006.

[4] A. A. Deo, R. J. Grill, K. M. Hasan, and P. A. Narayana, "In vivo serial diffusion tensor imaging of experimental spinal cord injury," $J$ Neurosci Res, vol. 83, pp. 801-10, Apr 2006.

[5] S. Wang, E. X. Wu, D. Qiu, L. H. Leung, H. F. Lau, and P. L. Khong, "Longitudinal diffusion tensor magnetic resonance imaging study of radiation-induced white matter damage in a rat model," Cancer Res, vol. 69, pp. 1190-8, Feb 12009.

[6] J. Yang and E. X. Wu, "Detection of cortical gray matter lesion in the late phase of mild hypoxic-ischemic injury by manganese-enhanced MRI," Neuroimage, vol. 39, pp. 669-79, Jan 152008.

[7] S. Wang, E. X. Wu, C. N. Tam, H. F. Lau, P. T. Cheung, and P. L. Khong, "Characterization of white matter injury in a hypoxic-ischemic neonatal rat model by diffusion tensor MRI," Stroke, vol. 39, pp. 2348-53, Aug 2008.

[8] E. S. Hui, M. M. Cheung, L. Qi, and E. X. Wu, "Towards better MR characterization of neural tissues using directional diffusion kurtosis analysis," Neuroimage, vol. 42, pp. 122-34, Aug 12008.

[9] M. M. Cheung, E. S. Hui, K. C. Chan, J. A. Helpern, L. Qi, and E. X. $\mathrm{Wu}$, "Does diffusion kurtosis imaging lead to better neural tissue characterization? A rodent brain maturation study," Neuroimage, vol. 45, pp. 386-92, Apr 12009.

[10] S. K. Song, S. W. Sun, M. J. Ramsbottom, C. Chang, J. Russell, and A. H. Cross, "Dysmyelination revealed through MRI as increased radial (but unchanged axial) diffusion of water," Neuroimage, vol. 17, pp. 1429-36, Nov 2002.

[11] J. H. Kim, M. D. Budde, H. F. Liang, R. S. Klein, J. H. Russell, A. H. Cross, and S. K. Song, "Detecting axon damage in spinal cord from a mouse model of multiple sclerosis," Neurobiol Dis, vol. 21, pp. 626-32, Mar 2006.

[12] M. D. Budde, J. H. Kim, H. F. Liang, R. E. Schmidt, J. H. Russell, A. H. Cross, and S. K. Song, "Toward accurate diagnosis of white matter pathology using diffusion tensor imaging," Magn Reson Med, vol. 57, pp. 688-95, Apr 2007.
[13] J. C. Ford, D. B. Hackney, D. C. Alsop, H. Jara, P. M. Joseph, C. M. Hand, and P. Black, "MRI characterization of diffusion coefficients in a rat spinal cord injury model," Magn Reson Med, vol. 31, pp. 488-94, May 1994.

[14] B. M. Ellingson, S. N. Kurpad, and B. D. Schmit, "Ex vivo diffusion tensor imaging and quantitative tractography of the rat spinal cord during long-term recovery from moderate spinal contusion," $J$ Magn Reson Imaging, vol. 28, pp. 1068-79, Nov 2008.

[15] S. Mi, B. Hu, K. Hahm, Y. Luo, E. S. Kam Hui, Q. Yuan, W. M. Wong, L. Wang, H. Su, T. H. Chu, J. Guo, W. Zhang, K. F. So, B. Pepinsky, Z. Shao, C. Graff, E. Garber, V. Jung, E. X. Wu, and W. Wu, "LINGO-1 antagonist promotes spinal cord remyelination and axonal integrity in MOG-induced experimental autoimmune encephalomyelitis," Nat Med, vol. 13, pp. 1228-33, Oct 2007.

[16] D. A. Fenyes and P. A. Narayana, "In vivo echo-planar imaging of rat spinal cord," Magn Reson Imaging, vol. 16, pp. 1249-55, Dec 1998

[17] B. M. Ellingson, S. N. Kurpad, S. J. Li, and B. D. Schmit, "In vivo diffusion tensor imaging of the rat spinal cord at 9.4T," J Magn Reson Imaging, vol. 27, pp. 634-42, Mar 2008.

[18] J. Gullapalli, J. Krejza, and E. D. Schwartz, "In vivo DTI evaluation of white matter tracts in rat spinal cord," J Magn Reson Imaging, vol. 24, pp. 231-4, Jul 2006.

[19] L. Concha, D. W. Gross, B. M. Wheatley, and C. Beaulieu, "Diffusion tensor imaging of time-dependent axonal and myelin degradation after corpus callosotomy in epilepsy patients," Neuroimage, vol. 32, pp. 1090-9, Sep 2006.

[20] O. al-Mefty, H. L. Harkey, I. Marawi, D. E. Haines, D. F. Peeler, H. I. Wilner, R. R. Smith, H. R. Holaday, J. L. Haining, W. F. Russell, and et al., "Experimental chronic compressive cervical myelopathy," $J$ Neurosurg, vol. 79, pp. 550-61, Oct 1993.

[21] S. K. Song, J. Yoshino, T. Q. Le, S. J. Lin, S. W. Sun, A. H. Cross, and R. C. Armstrong, "Demyelination increases radial diffusivity in corpus callosum of mouse brain," Neuroimage, vol. 26, pp. 132-40, May 15 2005.

[22] C. Beaulieu, "The basis of anisotropic water diffusion in the nervous system - a technical review," NMR Biomed, vol. 15, pp. 435-55, Nov-Dec 2002.

[23] T. Goto and Y. Hoshino, "Electrophysiological, histological, and behavioral studies in a cat with acute compression of the spinal cord," $J$ Orthop Sci, vol. 6, pp. 59-67, 2001.

[24] J. C. Falconer, S. J. Liu, R. A. Abbe, and P. A. Narayana, "Time dependence of $\mathrm{N}$-acetyl-aspartate, lactate, and pyruvate concentrations following spinal cord injury," J Neurochem, vol. 66, pp. 717-22, Feb 1996. 